\title{
A new subunit influenza vaccine: acceptability compared with standard vaccines and antigenicity in increasing dosage*
}

\author{
FREDERICK L. RUBEN \\ M.D. \\ GEORGE GEE JACKSON
M.D. \\ Department of Medicine, University Hospital, Abraham Lincoln School of Medicine \\ University of Illinois College of Medicine, Chicago, Illinois
}

\begin{abstract}
Summary
Subunit influenza vaccines have the advantage of purity and a broad dosage range. Clinical studies were done with a new subunit vaccine produced by detergent fractionation with tri-(n-butyl) phosphate (TNBP). Acceptability by the recipients was compared in volunteers given $400 \mathrm{CCA}$ units of $A_{2}$ and 300 of $B$ Influenza vaccine as either Sharples, zonal centrifuged, ether subunit, or TNBP subunit bivalent vaccines. When given subcutaneously, subunit vaccine caused only slightly less pain and erythema induration than zonal or Sharples vaccine. Compared with the i.m. route, the same vaccine given subcutaneously produced twice the local pain and eight times the erythema induration. Fever was infrequent and low grade by either route. HI antibody rises were good in all groups. Increasing dosages of TNBP vaccine up to 6400 CCA units of $A_{2}$ and 4800 of $B$ given i.m. as monovalent vaccine, produced no local and few febrile responses. An augmentation of the antibody titre occurred with the highest CCA unit dosages. Subunit vaccine given i.m. permitted administration of high CCA unit dosage beyond that accepted for standard vaccine without any increase in local reactions and with a measurable increase in antibody production and the induction of antibodies against related heterologous strains.
\end{abstract}

INFLUENZA virus vaccines have always been prepared from virus grown in embryonated eggs and traditionally consisted of inactivated whole virus. Some degree of purification of the virus from egg contaminants was accomplished by use of the Sharples international centrifuge. The end-product vaccine still contained a considerable amount of egg protein and the vaccines frequently produced local and febrile reactions. An important advance came with use of the zonal centrifuge to further purify the vaccine virus in a density gradient (Reimer et al., 1967). Another

\footnotetext{
* Taken from an article originally published in the Journal of Infectious Diseases (1972) 125, 656.

Requests for reprints: Frederick L. Ruben, Department of Medicine, Montefiore Hospital, 3459 Fifth Avenue, Pittsburgh, $\mathrm{Pa} .15213$.
}

advance came when the influenza virion was disrupted by treatment with ether. Vaccines prepared in this manner contained the envelope proteins (haemagglutinin and neuraminidase) of the virion separated from the nucleoproteins. Febrile responses were diminished and the antigenicity of the virus retained (Davenport et al., 1964).

Recently a new method for disrupting the influenza virion was reported by Neurath et al. $(1970,1971)$. A dispersant tri-( $n$-butyl) phosphate, or TNBP, was used to release the envelope proteins of the virus. Egg constituents were removed from the viral components by column separation. Some potential advantages of the method over disruption of the virus with ether are: that the TNBP-treated virus vaccine was less pyrogenic when given to rabbits, the virus was disrupted at very low $v / \mathrm{v}$ TNBP concentrations, and TNBP is non-volatile and, hence, safer for mass production techniques.

In clinical trials with TNBP-produced subunit vaccine, we have compared the local and systemic reactions and the immunologic response with other prototype influenza vaccines. With the new vaccine, high doses beyond those well-tolerated with conventional preparations also have been investigated.

\section{Materials and methods Vaccines}

Subunit tri-(n-butyl) phosphate vaccines were prepared by Wyeth Laboratories from egg grown $A_{2} /$ Aichi/2/68 and $B /$ Mass/3/66 influenza viruses. The final vaccine has essentially all of the original viral subunits, as well as a low level $(1 / 10,000)$ of formalin to inactivate any residual undisrupted virus. The standard formula (400 CC.A units $A_{2}$ and 300 CCA B per dose) vaccines was used. Three licensed vaccines (Sharples (Wyeth Conventional vaccine lot \#118201), zonal ultracentrifuged (Merck-SharpDohme vaccine lot $\$ 2126 \mathrm{M}$ ), and subunit ether (Parke-Davis vaccine lot $\$ 992598 \mathrm{C}$ )) were used for comparison.

\section{Population}

Healthy medical, nursing, and seminary students 
and new naval recruits participated on a voluntary basis. Their ages ranged predominantly from 18 to 25 years with a few older subjects. All were randomly assigned to vaccine groups and persons with allergy to eggs or egg-products were excluded.

\section{Immunization procedure}

Vaccines were given by the intradermal (i.d.), subcutaneous (s.c.) or intramuscular (i.m.) route; TNBP subunit vaccine was given by all three routes. All vaccines compared were bivalent and contained the same amount of viral haemagglutinin. Every local vaccination site was inspected at 24,48 , and when indicated, $72 \mathrm{hr}$. The maximum intensity of pain observed within the first $72 \mathrm{hr}$ was recorded on a scale of $0-4 ; 0$ meant no pain on palpation or motion of the injected arm and a rating of 4 was given for pain in the arm at rest. Oral temperatures were taken hourly for up to $8 \mathrm{hr}$ on the day of vaccination, and at 24 and $48 \mathrm{hr}$ after vaccination. Because it was not known whether adverse effects from increasing dosages of subunit TNBP vaccines would occur, the vaccines were given 'single blind', with the physician apprised of the dose given.

\section{Safety of the subunit TNBP vaccine}

The safety of the new vaccine was determined by running biochemical studies on the volunteers' sera for recognition of liver, renal, and haematopoietic toxicity. Sera from prevaccination, $48 \mathrm{hr}$, and 10 days postvaccination were tested. The test used was the SMA $12 / 60$ by Technicon Corporation, Tarrytown, New York, which included measurements of serum transaminase (SGOT), lactic dehydrogenase, alkaline phosphatase, creatine phosphokinase, calcium, phosphorous, total protein, albumin, cholesterol, uric acid, creatinine and total bilirubin.

\section{Clinical specimens}

Whole blood was drawn before immunization and at an average for each group of 23-31 days postvaccination. Nasal washings on selected volunteers were done on the same days the sera were collected.

\section{Haemagglunation inhibition $(H I)$ tests}

Sera were treated with receptor-destroying enzyme (RDE) (Microbiological Associates, Lot 3-3016 (Titre 1: 800)) of Vibrio cholera to remove non-specific inhibitors. HI testing was done by microtechnique (Sever, 1962). Heterologous haemagglutinins were obtained from the National Center for Disease Control, Atlanta, Georgia.

\section{Neutralization tests}

Sera were heat-inactivated at $56^{\circ} \mathrm{C}$ for $30 \mathrm{~min}$, and then serially diluted in monkey kidney main- tenance media at $\mathrm{pH} \mathrm{7.6}$. An equal volume, $0.4 \mathrm{ml}$ containing $100 \mathrm{TCID}_{50} / 0 \cdot 1 \mathrm{ml}$ of tissue culture adapted $\mathrm{A}_{2} /$ Aichi/2/68 or $\mathrm{B} / \mathrm{Mass} / 3 / 66$ virus was added and the mixture allowed to sit at room temperature for $1 \mathrm{hr}$, then $0.2 \mathrm{ml}$ of the mixture at each serum dilution was added to each of three tubes of monkey kidney cells in tissue culture. The cultures were incubated on a roller drum at $34^{\circ} \mathrm{C}$ for 3 days. At this time the replication of influenza virus was determined by the haemadsorption test with $0.5 \%$ guinea-pig erythrocytes.

Nasal washing specimens were lyophylized to dryness, reconstituted with $3.0 \mathrm{ml}$ saline, and tested for the presence of haemoglobin by Hematest (Ames Laboratories). All positive specimens were discarded. Aliquots of haemoglobin negative specimens were heat inactivated as above and handled exactly as serum for neutralization testing except that $10 \mathrm{TCID}_{50} / 0 \cdot 1 \mathrm{ml}$ was used as the virus inoculum and tubes were tested for haemadsorption after 4 days. A positive nasal washing neutralization test was defined as a four-fold rise in titre if there was a prevaccine titre, or any rise in titre if the prevaccine specimen at $1: 2$ dilution was negative. All paired nasal washings were run simultaneously.

\section{Complement fixation tests}

Paired serum specimens were tested using standard micro-titre complement fixation procedures (Sever, 1962) with overnight fixation at $4^{\circ} \mathrm{C}$ and two full units of complement. A commercial soluble (S) antigen of Influenza A and B was used.

\section{Results}

\section{Safety of the TNBP vaccine}

Two hundred and two sera representing sixty-seven volunteers were tested for abnormalities by the SMA $12 / 60$ automated test. None of the sera tested showed abnormalities either prior to receiving the vaccine, or at $48 \mathrm{hr}$ and $\mathbf{1 0}$ days after vaccination.

\section{Vaccines given at conventional dosage}

Figure 1 shows the effect of route of administration on the production of local and systemic sidereactions from the different influenza virus vaccines.

The three vaccines given s.c., Sharples, zonal and TNBP subunit, all caused maximal pain responses of an intensity over 2 . The vaccines given i.m., the subunit ether and subunit TNBP, had lower maximal pain responses. Erythema and induration at the local site which averaged $4-5 \mathrm{~cm}$ in diameter with vaccines given s.c., was hardly measurable in the i.m. vaccinated groups. Upper normal limits for fever were established in controls as $99.4^{\circ} \mathrm{F}$. Fever was minimal in all groups, both for the number of subjects who had fever and for the height of the temperature rise. 

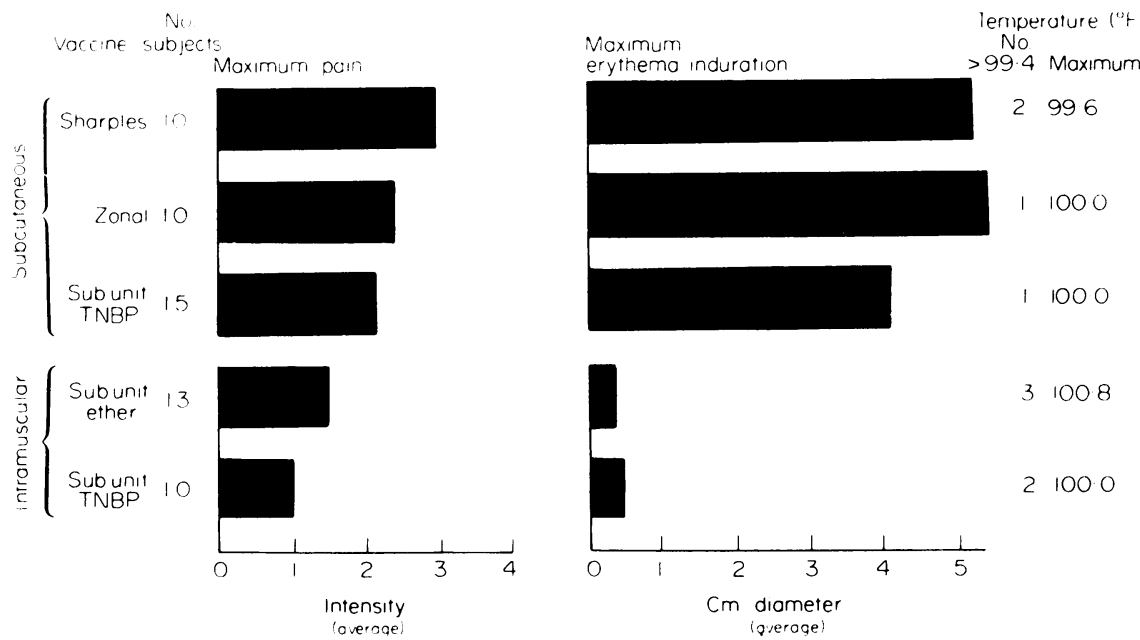

Fici. I. Local and systemic reactions following standard dosage influenza vaccines. All vaccines contain $400 \mathrm{CCA} \mathrm{A}_{2}$ and 300 CCA B.

TABLE I. HI antihody responses to all bivalent influenza vaccines

\begin{tabular}{|c|c|c|c|c|c|c|}
\hline \multirow{2}{*}{ Vaccine } & \multirow{2}{*}{$\begin{array}{l}\mathrm{CCA} \\
\mathrm{A}_{2} / \mathrm{B}\end{array}$} & \multirow{2}{*}{ Route } & \multicolumn{2}{|c|}{$\begin{array}{l}\text { Persons having four-fold rise } \\
\text { in HI antibodies total }\end{array}$} & \multicolumn{2}{|c|}{ Initial/final $\mathrm{HI}$ antibody status* } \\
\hline & & & $A_{2} /$ Aichi & B/Mass & $\mathrm{A}_{2} / \mathrm{Aichi}$ & B/Mass \\
\hline \multicolumn{7}{|l|}{ Subnit } \\
\hline TNBP & $40 / 30$ & i.d. & $9 / 9$ & 79 & $16: 167$ & $2 5 \longdiv { 2 3 9 }$ \\
\hline Sharples & $400 / 300$ & s.c. & $8 / 9$ & 59 & $11 / 126$ & $100 / 528$ \\
\hline Zonal & $400 / 300$ & s.c. & $9 / 10$ & $8 / 10$ & $36 / 214$ & $89 / 852$ \\
\hline \multicolumn{7}{|l|}{ Subunit } \\
\hline TNBP & $400 / 300$ & s.c. & 1114 & $1.3 / 14$ & 36247 & $89 / 1178$ \\
\hline \multicolumn{7}{|l|}{ Subunit } \\
\hline Ether & $400 / 300$ & i.m. & 1112 & 1112 & 85750 & 89511 \\
\hline \multicolumn{7}{|l|}{ Subunit } \\
\hline TNBP & $400 / 300$ & i.m. & $9 / 10$ & $9 / 10$ & $25 / 513$ & $200 / 1590$ \\
\hline \multicolumn{7}{|l|}{ Subunit } \\
\hline TNBP & $800 / 600$ & i.m. & $10 / 10$ & $8 / 10$ & $20 / 289$ & $250 / 1820$ \\
\hline \multicolumn{7}{|l|}{ Subunit } \\
\hline TNBP & $1600 / 1200$ & i.m. & $10 / 10$ & 45 & $16 / 390$ & 1502400 \\
\hline \multicolumn{7}{|l|}{ Subunit } \\
\hline TNBP & $3200 / 2400$ & i.m. & 1011 & 811 & $6 / 86$ & $25 / 303$ \\
\hline \multicolumn{7}{|l|}{ Subunit } \\
\hline TNBP & $6400 / 4800$ & $\mathrm{i} . \mathrm{m}$. & $9 / 9$ & $8 / 9$ & $4 / 258$ & 25698 \\
\hline
\end{tabular}

* (ieometric mean titre (reciprocal).

Table 1 gives the frequency of the $\mathrm{HI}$ antibody responses in all vaccine groups. In the groups given standard dose vaccine by s.c. or i.m. routes, four-fold rises over the pre-existing titres against the homologous strain of Influenza $A_{._{2}}$ occurred in all but seven of fifty-five subjects, and there were no significant differences among the vaccines or between the two routes. Six persons without initial antibody all showed seroconversion. The number of subjects with responses to Influenza $B$ at conventional dosage was comparable with that with $A_{2}$ but in the combined groups given subunit vaccine by either route, the seroconversion rate was greater, thirty-three of thirty-six, than with whole virus vaccine, thirteen of nineteen. The difference is of borderline statistical significance. The geometric mean titres pre- and postvaccine are also given in Table 1. Final titres against $A_{2}$ were higher in the i.m. groups, whereas against $B$, the highest titres were seen following TNBP vaccines. The average geometric fold rises in the serum titre of $\mathrm{HI}$ antibody against Influenza $\mathrm{A}$ or $\mathrm{B}$ as seen in Fig. 2 were similar following the different vaccines with one exception, the increment of the response to Influenza $A_{2}$ TNBP subunit vaccine given i.m. 


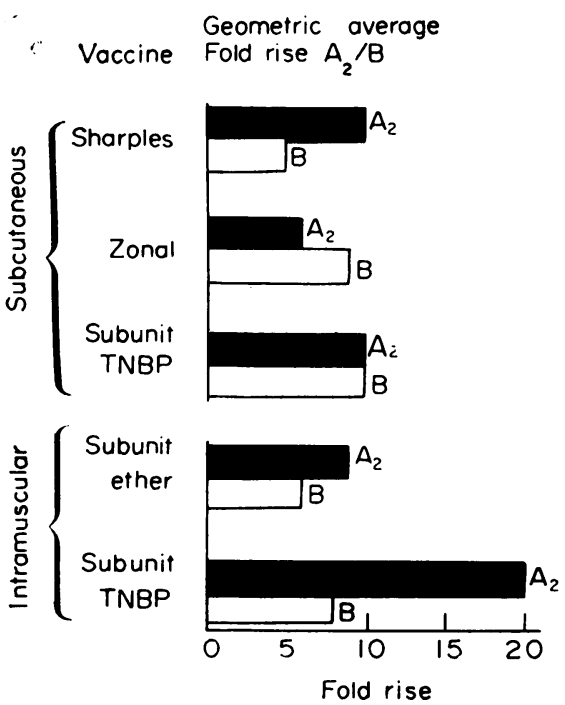

FIG. 2. HI antibody responses following standard dosage influenza vaccines.

which was double that observed in the other groups and is statistically significant $(P=<0.05)$.

An additional 200 subjects received TNBP subunit vaccine given i.m. and occasionally s.c. at standard dosage. The frequency of rises in different types or functions of antibodies in the serum and nasal washings of randomly selected samples from these subjects is shown in Fig. 3. HI antibody was the most sensitive indicator of seroconversion; responses in neutralizing antibody were nearly as frequent. Twelve of thirty sera were initially free of neutralizing antibody to $\mathrm{A}_{2}$, and eleven of these showed rises following vaccine. Eleven of thirty sera were free of neutralizing antibody to $B$ and seven of these showed rises following vaccine. Complement-fixing antibody conversions occurred in one-fifth to one-third of

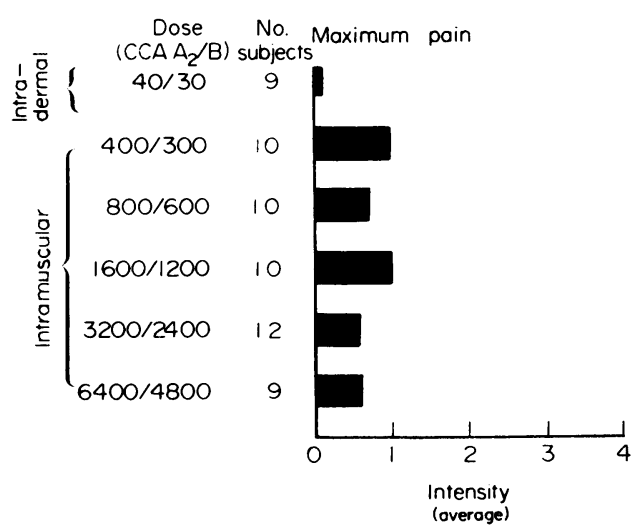

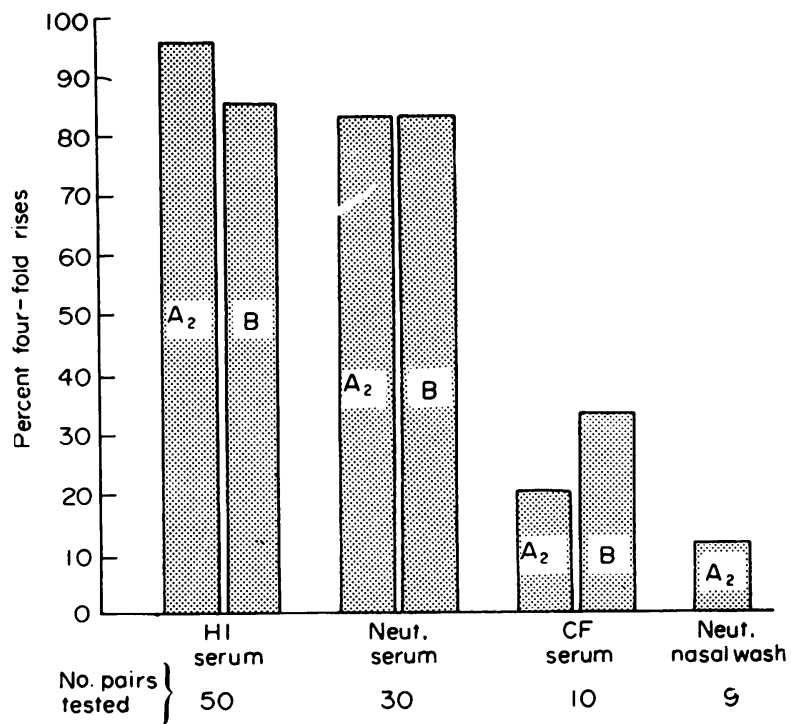

FIG. 3. Serum and secretory antibody responses following standard dosage TNBP subunit influenza vaccine. Number vaccinated-210.

vaccinated persons tested. Only one of nine nasal wash specimens tested for neutralizing antibody showed a rise in titre, and this went from less than 2 to a titre of 4 . IgA was present in all of the specimens studied at a concentration of $3-6.5 \mathrm{mg} / 100 \mathrm{ml}$ for $\rightleftharpoons$ the final reconstituted specimen.

\section{Vaccines given at increasing dosage}

Figure 4 shows the relative freedom from sidereactions with increasing doses of subunit vaccine given i.m. A small dose, $40 \mathrm{CCA}$ units of $\mathrm{A}_{2}$ and 30 CCA units of $B$ in a bivalent vaccine, given intradermally and shown for comparison, produced statistically significant erythema and induration,

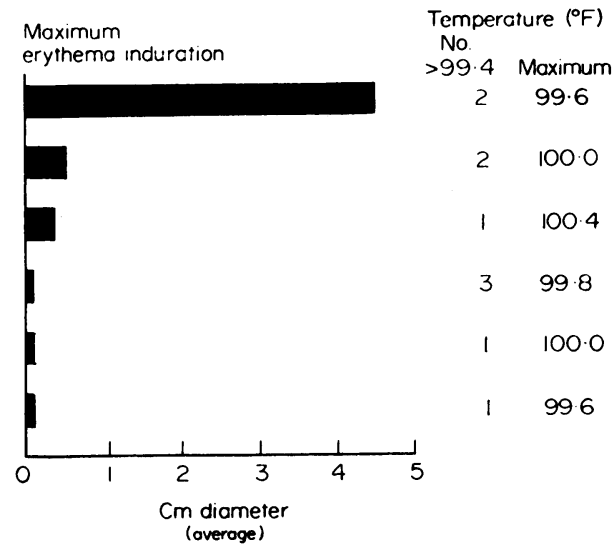

FIG. 4. Local and systemic reactions following increasing dosages of TNBP subunit influenza vaccine. 
whereas 160 times the dose given by i.m. injection produced virtually no local reactions or statistically significant temperature elevations.

As seen in Table 1 , an antibody rise against $A_{2}$ was elicited in all but two of fifty persons given $400 \mathrm{CCA}$ units or more of $A_{2}$ subunit vaccine i.m. This included seventeen persons without initial antibody to Influenza $\mathrm{A}_{2}$ and one without antibody to $\mathrm{B}$. Onetenth that dose intradermally also stimulated a rise in all of nine persons. Hence, the frequency of seroconversion was the same with any of these doses. The geometric mean antibody titres following increasing dosages of TNBP vaccines showed no correlation with the dosage either for $A_{2}$ or $B$. Figure 5 shows the magnitude of the increment in HI antibody expressed as geometric average fold rises. All were nearly comparable except for the highest dosage. At $6400 \mathrm{CCA}$ units of $A_{2}$ (with 4800 CCA units of B) a striking increase in antibody occurred relative to the other dosages which was statistically significant $(P=<0.05)$. Neutralizing antibody titres are not illustrated, but in the highest dose vaccine group they were similarly increased. None of the doses of Influenza B evoked an antibody response in all of the recipients; seroconversion occurred in only thirty-seven of forty-five persons given doses from 300 to 4800 CCA units. Seven of nine responded to $30 \mathrm{CCA}$ units intradermally. Higher dose elicited a greater increment in the magnitude of the antibody response, but the dose effect was significant $(P=<0.05)$ only at the highest dosage.

Sera from 10 days postvaccination are compared with the final specimens in Fig. 6. With the doses of $A_{2}$ vaccine, an abrupt break in the curve is seen

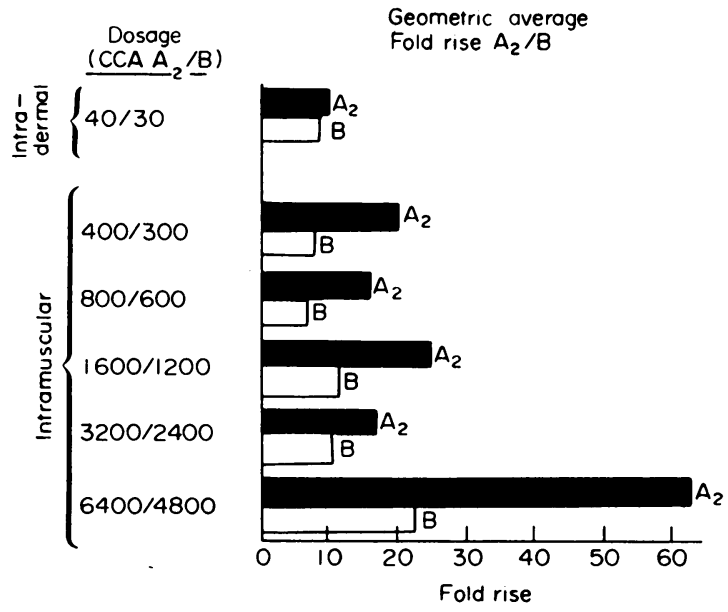

FIG. 5. HI antibody responses following increasing dosages of TNBP subunit influenza vaccine. showing the marked increment in titre of antibody achieved following a dose of 6400 CCA units. A similar but less striking effect is seen with Influenza B vaccine at a dose of 4800 . Another feature of the curves is that with doses up to $1600 \mathrm{CCA}$ units of $\mathrm{A}_{2}$, the maximum antibody response was not attained until 3-4 weeks after vaccination (the final sera), whereas larger doses of vaccine produced a peak titre earlier with an appreciable decrease after 3-4 weeks.

In Table 2 are the results of tests for $\mathrm{HI}$ antibody against heterologous strains of influenza virus. Sera from vaccinees receiving the standard dose, $400 \mathrm{CCA}$ units of $A_{2}$ and 300 CCA units of $B$ subunit vaccine were compared with sera from vaccinees receiving the highest dosage, which was sixteen times greater. Testing was done with haemagglutinins from three characteristic isolates of $A_{2}$ from earlier periods, two strains of $A_{1}$, the human prototype $A$ (PR 8), and A (Swine); also, two earlier strains of Influenza B were used. The paired sera used in these tests had shown rises to both $\mathrm{A}_{2} / \mathrm{Aichi}$ and $\mathrm{B} /$ Mass, the vaccine

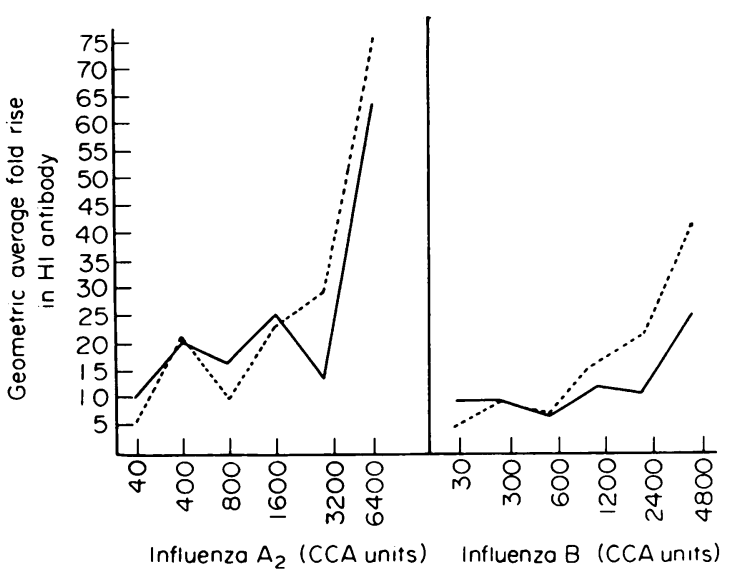

FIG. 6. Early against final $\mathrm{HI}$ antibody responses to increasing dosages of TNBP subunit influenza vaccine. ,-- 10 day specimen; $\_$, final specimen $(23-31$ days).

TABLE 2. Heterologous influenza HI antibody responses

\begin{tabular}{ccccc}
\hline & \multicolumn{4}{c}{ No. heterologous rises/no. tested } \\
\cline { 2 - 5 } Vaccine subunit & $\mathrm{A}_{2}{ }^{*}$ & $\mathrm{~A}_{1}{ }^{*}$ & $\mathrm{~A}_{0}{ }^{*}$ & $\mathrm{~B}^{*}$ \\
\hline $400 / 300$ & $0 / 2$ & $0 / 4$ & $0 / 4$ & $3 / 4$ \\
$6400 / 4800$ & $2 / 4$ & $3 / 4$ & $0 / 6$ & $3 / 4$ \\
\hline
\end{tabular}

* Heterologous strains tested: $A_{2} ; 134 / 64$, Jap/305/57, Jap/107/62: $A_{1}$; Denver/57, FM-1/47: $A_{0}$; Swine/31, PR8/34: B; Great Lakes/54, Lee/40. 
strains. Although the number of observations is small, the lower dosage vaccine was not observed to induce any rises in antibody that inhibited earlier heterologous $A_{2}$ or $A_{1}$ strains. In contrast, a fourfold or greater increase in $\mathrm{HI}$ antibody against the previously prevalent $A_{2}$ and $A_{1}$ strains was demonstrated in five of eight pairs of sera tested from persons given the high dose of subunit vaccine. None of the persons had lived during the period when the prototype Influenza A strains were prevalent, and none of the sera showed the development of inhibiting antibody against these strains. Heterologous antibody responses to the earlier B strains occurred with Influenza B/Mass vaccine at either low or high dosage.

\section{Discussion}

In this clinical study, a subunit vaccine prepared by a new treatment of virus harvests with a detergent TNBP and separation of the viral components on a porous column (Neurath et al., 1970, 1971) was compared with other available vaccines. Also of interest was the new opportunity afforded by the acceptability of the subunit vaccine to explore the response over a broad range of doses up to sixteen times the standard recommended dose.

For Influenza $A_{2}$ in the standard dose of $400 \mathrm{CCA}$ units, the subunit vaccine had no apparent advantage over whole virus preparations with regard to the number of people in whom an antibody response was elicited. In the case of Influenza B using 300 CCA units, the subunit preparation was advantageous, but more extensive studies are necessary to make such a conclusion final. Although the magnitude of the rise following i.m. administration of standard dose TNBP subunit $A_{2}$ vaccine was greater than those following other vaccines, the result could be from differences in the average pre-vaccine antibody titre.

The influence of route administration, and particularly of the i.m. route, on acceptability of influenza vaccines has not received great attention in the literature. Route has been shown to influence antibody responses, particularly with low dosages (Tauraso et al., 1969). The subunit TNBP vaccine, when given intradermally or subcutaneously, caused reactions comparable with those produced by the Sharples and zonal vaccines. These were typical reactions of delayed hypersensitivity indicating prior sensitization of the recipients to influenza virus antigens or else local cellular recruitment with the liberation of vasoactive substances at the site of antigen injection. Studies to be reported elsewhere suggest the former and also suggest that an increase in the number of specifically sensitized lymphocytes occurred in the blood after vaccination. When the route was switched to i.m., virtually no reactions were noted with the subunit TNBP vaccine, even including the highest CCA unit dosages given. This reduction in local reactions suggests that the vaccine may have disseminated rapidly from the site of injection, which could result in diminished antigenicity. No data are available to know whether there was a decreased stimulus to sensitization of T-lymphocytes, but there was no decrease in antibody response following the i.m. injection of a standard dose of subunit vaccine. In fact, the geometric mean response of antibody titre to the same dose of TNBP subunit Influenza $A_{2}$ vaccine was better by the i.m. than the s.c. route; the average titre rises were 25-513 against 36-247 respectively. For Influenza B also, a higher mean peak titre was reached after i.m. than s.c. vaccine (1500 against 1180 ), but the initial mean titre of the group also was higher (200 against 89); therefore, no difference can be assumed.

The observation that i.m. injection of subunit vaccine eliminated the local painful reactions and did not decrease the antibody response, permitted investigation of the response to increasing dosages of vaccine. Mostow et al. (1969), in one study, compared high (3000 CCA units) dosages of zonal centrifuged vaccines given subcutaneously. They found a two- to three-fold greater incidence of adverse reactions and a ten-fold increase in antibody titre following the higher dose. Phillips et al. (1970 studied zonal centrifuged vaccines in a dose range of 200-800 CCA units and reported loca reactions in $19 \%$ of the volunteers receiving vaccine subcutaneously. They also showed geometric mean titres which increased with the increments in dosage. Mostow et al. (1970) in a separate study observed erythema of greater than $5 \mathrm{~cm}$ and induration of at least $2 \mathrm{~cm}$ in over $40 \%$ of vaccinees from $4800 \mathrm{CCA}$ units of a zonal centrifuged Influenza $A_{2}$ vaccine given subcutaneously. A dose-response curve was demonstrated for HI serum antibody showing the highest titres following maximum CCA unit dosages. Heterologous A antibody formation was also studied and found following the higher dosages of vaccine. In our study, large doses of TNBP subunit vaccine given i.m. caused no intolerance, but increased the response only modestly, up to twenty-five fold for $A_{2}$ and up to twelve-fold for $B$, until the highest doses (6400 CCA units of $A_{2}$ and 4800 CCA units of B) were used.

The types of antibody responses following TNBP subunit vaccine showed that rises in neutralizing antibody paralleled those in HI antibody. The occurrence of some rises in complement-fixing antibody against soluble (S) antigens of Influenza $\mathrm{A}$ and B is of interest. It is generally accepted that disease almost uniformly induced anti-(S) rises in humans. The acquisition of these antibodies following vaccination has not frequently been tested. Since 
there was no clinical illness in any of the volunteers during the study, we assume that the anti-(S) rises were vaccine-induced, and that the vaccine contains some viral nucleoprotein. The rises confirm the observation of Lief, Henle \& Schrack (1960) that inactivated vaccines can induce such rises. In another report, data were published which showed that the standard doses of TNBP subunit vaccine, and all of the other preparations, were effective in stimulating rises in anti-neuraminidase antibody. The magnitude of the response in general paralleled that of the $\mathrm{HI}$ antibody (Neurath et al., 1971). The failure of the TNBP vaccine in standard dosage to induce neutralizing activity in the nasal washings was disappointing. Previous observations using standard vaccine have given varied results (Phillips et al., 1970; Mostow et al., 1970; Fulk et al., 1969). Intranasal administration of inactivated vaccine can sometimes elicit nasal antibody, but the production of local immunity of the respiratory tract is one of the potential advantages of live virus vaccines under development.

The serum response to TNBP subunit vaccine was rapid, especially when large CCA unit doses were given. These must be assumed to be secondary or booster responses considering that most subjects had pre-existing antibody. The few persons who had no detectable antibody against the vaccine strain, however, also showed prompt seroconversion after subunit vaccine.

It is hoped that the large doses of vaccine which produced many fold increases in the titre of antibodies might also result in a persistent protective level. Data in this respect are not yet available, but the recognition of a decline in the antibody titre three to four weeks after vaccination suggests an active feedback mechanism that suppressed continued antibody formation in the presence of high titres in the serum. Feedback suppression seems a more likely explanation for the fall than the evolution from IgM to IgG antibodies. If so, it may be necessary regardless of the dose administered to consider combining the subunit vaccine with a respository adjuvant if persistent antibody stimulation and long-term protection are to be achieved without frequent revaccination.

Heterologous antibody responses following subunit vaccine administration have been clearly demonstrated in animals (Fazekas, 1969). Such responses in humans following zonal centrifuged vaccines are reported in the study of Mostow et al. (1970). In our study, heterologous antibody responses to previously encountered Influenza $A$ and interrelated $B$ strains were demonstrated following subunit TNBP vaccine. Among strains of Influenza A, heterologous antibodies were produced when a large dose of vaccine was given and were not induced by the standard dose of 400 CCA units. It seems unlikely that small increments in CCA unitage of Influenza $\mathrm{A}$ vaccine will be useful for heterologous antibody induction. On the other hand, standard doses of 300 CCA units of Influenza B vaccine readily produced inhibitory antibody against the heterologous strains isolated in earlier periods. These and other biologic differences between Influenza $A$ and $B$ viruses make it prudent to consider the vaccine requirements for each independently as new vaccines are formulated with the possibility of broad variations in the range of dosage. If production of antibody against related heterologous strains is a goal, a reasonable formula, according to our data, might be 300 CCA units of Influenza $B$ with 6400 CCA units of A.

The ability to give relatively large doses of subunit vaccine without producing undesirable side-reactions also permits re-inquiry about the theoretical advantages of a polyvalent vaccine. If adequate doses of antigens from past strains of Influenza $A$ can be included without compromising the effective, protective dose of antigens from recent strains, the likelihood of prophylaxis against more of the natural recombinants of influenza viruses seems probable. A new subunit vaccine appears at least to offer the possibility for such a study.

\section{Acknowledgments}

The authors acknowledge the following with appreciation for their help with this study: Dr Robert L. Muldoon, Mary Rubenis, Linda Mezny and Valentina Lolans of the University of Illinois; Captain Robert O. Peckinpaugh and Commander Robert Mammon of the Great Lakes Naval Training Center, Great Lakes, Illinois; Dr Ben Rubin, Dr Robert Neurath, and Dr Sam Wong of Wyeth Laboratories, Incorporated.

Supported in part by U.S. Public Health Service, Research Grant AI4059 and Training Grant AI208 and by Wyeth Laboratories, Inc., Philadephia, Pa.

\section{References}

Davenport, F.M., Hennessy, A.V., Brandon, F.M., Webster, R.G., Barrett, C.D. \& Lease, G.O. (1964). Comparison of serologic and febrile responses in humans to vaccination with Influenza $A$ viruses on their haemagglutinins. Journal of Laboratory and Clinical Medicine. 63, 5 .

FAZEKAS DE ST GRoth, S. (1969) The antigenic subunits of influenza viruses. Journal of Immunology, 103, 1107.

FulK, R.V., Fedson, D.S., Huber, M.A., FitzPatrick, R., Howar, B.F. \& Kasel, J.A. (1969) Antibody responses in children and elderly persons following local or parenteral administration of an inactivated influenza virus vaccine, $\mathrm{A}_{2}$ Hong Kong/68 variant. Journal of Immunology, 102, 1102.

Lief, F.S., Henle, W. \& Schrack, W.D. (1960) Antigenic analysis of influenza virus by complement fixation. Journal of Immunology, 85, 483.

Mostow, S.R., Schoenbaum, S.C., Dowdle, W.R., ColeMAN, M.T. \& KAYE, H.S. (1969) Studies with inactivated influenza vaccines purified by zonal centrifugation. Bulletin of the World Health Organization, 41, 525. 
Mostow, S.R., Schoenbaum, S.C., Dowdle, W.R., ColeMAN, M .T. \& KAYE, H.S. (1970) Studies on inactivated influenza vaccines. American Journal of Epidemiology, 92, 248.

Neurath, A.R., Stasny, J.T., Rubin, B.A., Fontes, A.K. Pierzchala, W.A., Wiener, F.P. \& Hartzell, R.W. (1970) The effect of nonaqueous solvents on the quarternary structure of viruses: properties of haemagglutinins obtained by disruption of influenza viruses with tri-( $n$-butyl) phosphate. Microbios, 7-8, 209.

Neurath, A.R., Rubin, B.A., Sillaman, J. \& Tint, H. (1971) The effect of nonaqueous solvents on the quarternary concentration, purification and disruption of influenza viruses. Microbios, 14, 145.

Neurath, A.R., Ruben, F.L., Rubin, B.A., Hartzell, R.W. \& Wong, S. (1971) Antibody response in humans to neuraminidase of $A_{2} / A i c h i / 68$ and $B / M a s s / 66$ influenza viruses. Proceedings of the Society for Experimental Biology and Medicine, 138, 47.
Phillips, C.A., Forsyth, B.R., Chirstman, W.A., Gump, D.W., Whorton, E.B., Rogers, I. \& Rubin, A. (1970) Purified influenza vaccine: clinical and serologic responses to varying dozes and different routes of immunization. Journal of Infectious Diseases, 122, 26.

Reimer, C.B., Baker, R.S., Vanfrank, R.M., Newlin, T.E., Cline, G.B. \& Anderson, N.G. (1967) Purification of large quantities of influenza virus by density gradient centrifugation. Journal of Virology, 1, 1207.

SEVER, J.L. (1962) Application of a microtechnique to viral serological investigations. Journal of Immunology, 88, 320.

Tauraso, N.M., Gleckman, R., Pedreira, F.A., Sabbaj, J., YAHWAK, R.\& MADOFF, M.A. (1969) Effect of dosage and route of inoculation upon antigenicity of inactivated influenza virus vaccine (Hong Kong strain) in man. Bulletin of the World Health Organization, 41, 507. 